\title{
Pathology comparative study on the characteristic CT signs in solid pseudopapillary neoplasm of the pancreas
}

\author{
XI BO FU ${ }^{1}$, ZHI QIANG HAO ${ }^{1}$, JIN YUN HE ${ }^{2}$, HAI SHANG ${ }^{1}$, QING CAI FU ${ }^{1}$, \\ XIANG DONG HUA ${ }^{1}$, YE FU LIU ${ }^{1}$ and JIE LIN ${ }^{1}$ \\ ${ }^{1}$ Department of Hepatobiliary Surgery, Liaoning Cancer Hospital, Shenyang, Liaoning 110042; \\ ${ }^{2}$ Department of General Surgery, Liaohe Oil Field General Hospital, Panjin, Liaoning 124010, P.R. China
}

Received June 16, 2015; Accepted August 30, 2016

DOI: $10.3892 /$ etm.2017.4382

\begin{abstract}
In order to analyze characteristic CT signs in the solid pseudopapillary tumor of the pancreas, a retrospective analysis was conducted on 49 patients with pseudopapillary tumor of the pancreas who where treated in Liaoning Cancer Hospital. All of the patients were confirmed by pathology, CT signs were analyzed and a pathology contrast was conducted. Furthermore, all cases had single lesions; 7 cases in the pancreatic head, 23 cases in the pancreatic body, 15 cases in the pancreatic body-tail and 4 cases in the pancreatic tail. The boundaries of the lesions were clear and the tumors, which may outline the pancreas, were composed of solid and polycystic parts. In addition, calcifications could be observed in the lesions and CT results revealed varying degrees of contrast enhancement of the solid components in the arterial phase, as well as a gradual contrast enhancement in the venous and delayed phase. Enhancement of capsule could be observed, and the enhancement region was observed in the solid part, no enhancement in cystic part.. In conclusion, CT manifestations of solid pseudopapillary tumors of the pancreas are specific, which is helpful to the diagnosis.
\end{abstract}

\section{Introduction}

Solid pseudopapillary tumor of the pancreas (SPTP) is a rare tumor that is benign or low-grade malignant, with benign or malignant potential, and a low incidence rate, accounting for $0.2-2.7 \%$ of the total pancreatic tumors (1). Since it has no characteristic clinical manifestations and a low incidence rate, the preoperative diagnosis rate is low. Furthermore, the treatment principle is different from other pancreatic tumors and an improvement of the preoperative diagnosis rate is important.

Correspondence to: Dr Xi Bo Fu, Department of Hepatobiliary Surgery, Liaoning Cancer Hospital, 44 Xiaoheyan Road, Shenyang, Liaoning 110042, P.R. China

E-mail: fuxibodr@126.com

Key words: solid pseudopapillary tumor of the pancreas, tomography, X-ray computed, diagnosis
SPTP is known to occur preferentially in young females and has a favorable prognosis (2-4). The characteristic imaging features of SPTPs include encapsulation, solid and cystic components and peripheral calcification (5-7). Furthermore, there exists no consensus on a common location of the tumor within the pancreas and they usually affect young women at an average age of 28 years old with a female:male ratio of 10:1 (8). However, sporadic rare cases in males and in the elderly have been reported. The tumor has a low-grade malignant potential and tends to have a favorable prognosis following resection, even in the presence of metastatic disease of up to $20 \%$ of cases (8). The overall 5-year survival exceeds $90 \%$, including patients with metastatic disease (9). Males, elderly, histopathologically atypical tumors and incomplete resections have been associated with a higher risk of recurrence and mortality (9).

To date, enhanced computed tomography (CT) has become a common examination method for pancreatic diseases, and understanding the enhanced characteristic CT signs of SPTP has important clinical significance. Therefore, the present study summarized the CT signs of 49 cases who received surgery in Liaoning Cancer Hospital (Shenyang, China) between January 2002 and May 2014, and then compared these with the pathological examination.

\section{Materials and methods}

Clinical data. A total of 49 cases were collected, including 42 female cases and 7 male cases, between 14-60 years old, and with a median age of 30.4 years. Among the cases, 18 cases had no obvious symptoms, and 31 cases had clinical symptoms; 15 cases had epigastric pain, 9 cases had epigastric discomfort, 6 cases had abdominal distension, 2 cases had fatigue, 4 cases had jaundice, 4 cases had back discomfort and 2 cases had fever. A number of cases had a combination of the symptoms described above. Among the cases, 4 cases had palpable abdominal mass and 2 cases had abdominal tenderness, while other cases had no positive abdominal signs.

Methods. In the present study, the primary radiological image quality and scanning time of 12 cases met the requirements, and the remaining 37 cases received a repeated CT scan. A Lightspeed 16-Slice Spiral CT (GE Healthcare Life Sciences, Chalfont, UK) was used with a slice thickness of $5 \mathrm{~mm}$, an 
interval of $5 \mathrm{~mm}$, thickness of $3 \mathrm{~mm}$ and an interval of $3 \mathrm{~mm}$. The scanning parameters were $120 \mathrm{kV}$ and $140 \mathrm{~mA}$. All cases were examined with a plain and dynamic enhanced scan. The patients were fasting for $\geq 8 \mathrm{~h}$, then firstly received a pancreatic plain scan, and then hepatic arterial phase, portal venous phase and delayed phase enhanced scanning were conducted. The contrast agent used was Omnipaque $(300 \mathrm{mg}(\mathrm{I}) / 100 \mathrm{ml})$, and it was injected with an elbow vein high pressure syringe at a dose of 90-100 ml. In addition, the injection rate was 2-3 $\mathrm{ml} / \mathrm{sec}, 20-25 \mathrm{sec}$ in the arterial phase and 60-70 sec in the portal venous phase. A number of cases received a delayed scan with a delay time of $120 \mathrm{sec}$, and lasted for $10 \mathrm{~min}$. The patients in this group did not receive a conventional revascularization check.

All the patients were treated with open surgery, including duodenum preserving pancreatic head resection in 7 cases, distal pancreatectomy splenectomy in 21 cases, simple distal pancreatectomy in 11 cases, pancreatic tail resection in 4 cases, middle pancreatectomy and distal pancreatic enteric anastomosis in 2 cases, local tumor resection in 3 cases, and local tumor resection and pancreaticojejunostomy in 1 case. Among them, 21 cases received lymph node dissection.

\section{Results}

Specimen staining. Collected the specimens from the part of lesions, and fixed with $10 \%$ neutral formalin, embedded with paraffin routinely, and sliced. Slices were treated as follows: Xylene (I), 5 min; xylene (II), 5 min; 100\% ethanol, 2 min; 95\% ethanol, $1 \mathrm{~min}$; $80 \%$ ethanol, $1 \mathrm{~min}$; 75\% ethanol, $1 \mathrm{~min}$; and distilled water, $2 \mathrm{~min}$. Hematoxylin staining for 5-20 min, then washed with water, and differentiated with ethanol hydrochloride $30 \mathrm{sec}$, then soaked in water for $15 \mathrm{~min}$. The slices were placed in the eosin dye for $2 \mathrm{~min}$, washed with water, and subjected conventional dehydration, clarified and sealed as follows: 95\% Ethanol (I), $1 \mathrm{~min}$; 95\% ethanol (II), $1 \mathrm{~min}$; 100\% ethanol (I), $1 \mathrm{~min}$; 100\% ethanol (II), $1 \mathrm{~min}$; xylene phenol (3:1), $1 \mathrm{~min}$; xylene (I) $1 \mathrm{~min}$; diphenyl (II), $1 \mathrm{~min}$; and neutral resin sealing. Sections were then observed under a microscope.

Lesion characteristics. All cases had single lesions. In total, 7 cases had lesions at the head of the pancreas, 23 cases had lesions at the pancreatic body, 15 cases had lesions at pancreatic body-tail, and 4 cases had the lesions at pancreatic tail. The maximum diameter of the lesions was $1.5-15.5 \mathrm{~cm}$ with an average of $5.8 \mathrm{~cm}$, and the lesions were often highlighted from the pancreatic contour and grew to the peritoneal cavity or retroperitoneal space. No pancreatic body and tail atrophy, in addition to dilation of the pancreatic duct, was observed. Furthermore, plain scanning revealed that there was a low mixed density, and the majority of the lesions had a clear boundary. In total, 4 cases had an unclear boundary and no obvious capsule, and 3 cases had an incomplete capsule. There were both cystic and solid parts in the lesions, and these accounted for different proportions. The main performance was cystic in 14 cases, solid in 13 cases, equal component of cystic and solid in 18 cases and completely solid in 4 cases. A total of 11 cases had the lesion combined with calcification, and the calcification lesions were often located at the edge and showed a linear arc or multiple punctate calcification.

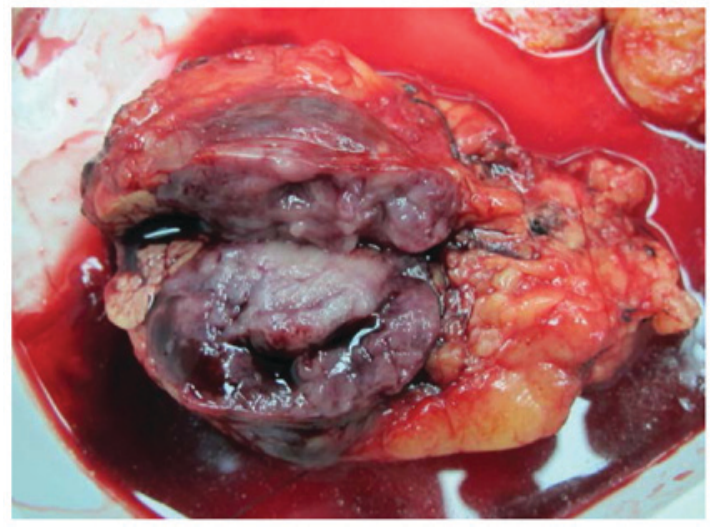

Figure 1. Specimen showing necrosis, hemorrhage and cystic degeneration.

Furthermore, calcification in 3 cases was located at the center of the lesion, and demonstrated an irregular annular or scattered punctate. Enhanced scanning revealed that the solid part of the arterial phase showed mild to moderate enhancement, the portal venous phase and delayed phase had a continuous enhancement and the tumor enhancement was higher than that of the arterial phase. The enhancement degree of the lesion was close to or lower than that of normal pancreatic tissue and the tumor enhancement was gradually extended from the peripheral to the center. In the present study, no distant metastasis or lymph node metastasis were observed.

All patients received a lesion resection, and there were no lesions invading the surrounding organs, tissue or blood vessels. Meanwhile, all cases were pathologically confirmed as SPTP, and all the patients had single lesions without distant or lymph node metastasis. The boundary of lesions was clear with a thick fibrous capsule outside the lesions, and had a clear boundary with the pancreas and other surrounding tissue. Furthermore, necrosis, hemorrhage and cystic degeneration could be observed on the lesion (Fig. 1). Microscopic observation demonstrated abundant cytoplasm, red staining, and same size and round or oval nuclei tumor cells without a rare nuclear division (Fig. 2). Furthermore, tumor cells were solid sheets or papillary arranged around the fibrovascular axis (Figs. 2 and 3). Microscope observation demonstrated a capsular invasion observed in 9 cases.

Patient outcome. All the patients were followed-up after surgery for a period of 1 month to 5 years (median follow-up, 37.4 months). A total of 3 cases were lost (the lost time was between 6,25 and 54 months after surgery), and no patients had a recurrence or transfer during the follow-up period.

\section{Discussion}

SPTP is a rare type of tumor with a low malignant potential. In 1959, Frantzs initially described its pathological features and named them as Frantzs tumors (10). Due to the shortage of characteristic clinical manifestations, the incidence rate is not high, and there is a lack of awareness in clinicians. According to their morphological characteristics, several descriptive names are used in the literature, including pancreas papillary epithelial tumors, cystic and solid tumors of the pancreas, solid epithelial tumors of the pancreas, papillary cystic tumors and 
pancreatic cystic papillary epithelial tumors (11). In 1996, the World Health Organization renamed the tumor as SPT and reclassified it as SPTP (12).

SPTP has a lower age of onset and is more common in females than in males $(13,14)$. In the present study, the median age of the patients was 30.4 years old, and the ratio of males to females was 1:6. Surgery is an effective treatment method and patients can often survive for a long time following surgery. Due to the biological characteristics of SPTP, distant and lymph node metastasis are rare, therefore, expanded tumor resection and conventional lymph node dissection should not be conducted, otherwise it will increase the surgical complications and reduce the quality of life following surgery while do nothing to improve the survival rates $(15,16)$. In the present study, a CT examination demonstrated no distant and lymph node metastasis. Lymph node dissection was conducted on 21 patients, and postoperative pathological examination revealed no lymph node metastasis. Thus, the correct preoperative diagnosis of SPTP in particular is necessary. Currently, the popularization of multislice spiral CT and the development of examination techniques and methods make it possible.

The CT observations and pathological features of SPTP are closely correlated. SPTP in cystic and solid tumors, as well as the ratio and the components of cystic and solid tumors, may affect CT imaging. In the present study, cystic and solid parts were identified in the lesions that account for different proportions (Figs. 4 and 5). This can be expressed as cystic, solid and equal components of cystic and solid parts. In addition, a number of researchers believe that larger tumors have a high probability of cystic degeneration $(5,6)$. Baek et al $(6)$ reported that small SPTP (diameter, $<3 \mathrm{~cm}$ ) was primarily expressed as a solid. The 4 cases in the present study were expressed as completely solid, and the lesion diameter was $<3 \mathrm{~cm}$, suggesting that there is a certain correlation between the tumor size and component ratio of cystic and solid parts. Furthermore, the preoperative and postoperative pathological examination demonstrated no tumor invasion to the surrounding blood vessels and organs, therefore, inspection technology of vascular reconstruction was not necessary for SPTPs.

SPTP lesions are often located outside the pancreatic contour, and grow to the abdominal and retroperitoneal relative emptiness. Furthermore, the majority have no pancreatic duct expansion since the tumor mostly reveals expansive growth and the lesion boundary is clear. In the present study, a tumor with a cystic component demonstrated low density, and a tumor with solid components revealed an uneven density, which may be correlated with tumor hemorrhage and necrosis (Fig. 5). Furthermore, the hemorrhage and necrosis component could be observed in the solid tumor of the postoperative section (Fig. 1). Calcification was revealed in part lesions and Buetow et al (17) identified that calcification could be observed in $30.8 \%$ of the tumors. In addition, the calcification located in the edge of the lesions was mostly calcified capsule, whereas the calcification located on the solid components was caused by intralesional fibrous connective tissue degeneration. Histopathologically, SPTP is classically defined as a large and encapsulated mass composed of a mixture of cystic and solid areas. Intratumoral hemorrhage is frequent, and calcifications have been reported in $\leq 30 \%$ of cases $(7,18)$.

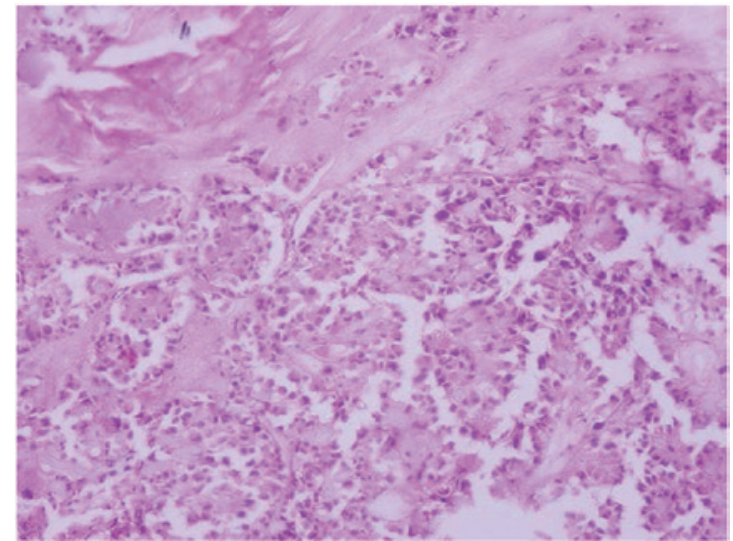

Figure 2. Microscopic observation of a solid pseudopapillary tumor of the pancreas. Tumor tissues are composed by multifocal fake papillary and hardening stroma with stromal mucoid degeneration. (hematoxylin-eosin staining; magnification, $\mathrm{x} 200$ ).

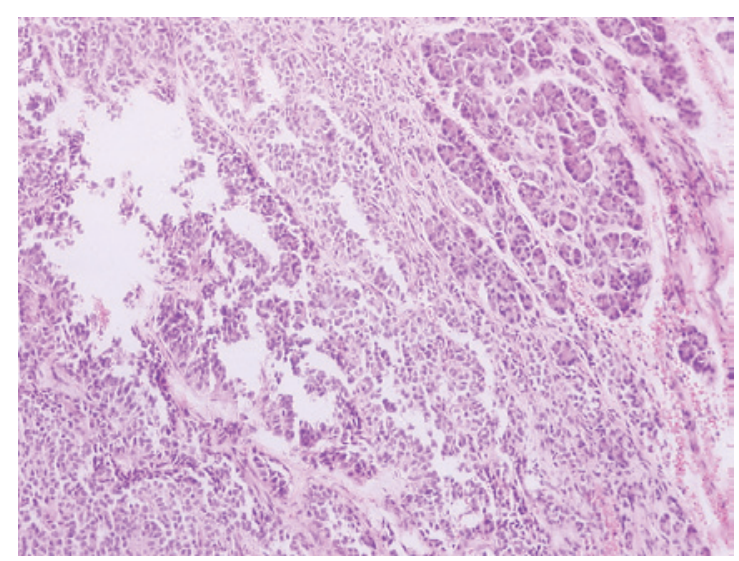

Figure 3. Microscopic observation of solid pseudopapillary tumor of the pancreas. The tumor tissues are composed by consistent solid nests tumor cells, and the fake papillary structure could be observed (hematoxylin-eosin staining, x200)

Although SPTP is considered to be an indolent lesion with a low malignant potential and a favorable prognosis following surgical resection, a number of cases of locally infiltrating and metastatic varieties and post-surgical recurrences have been reported (19).

In the present study, 11 cases had bleeding calcification, and the calcification lesions were located in the edge of each lesion. Furthermore, they were expressed as a linear arc or multiple punctate calcification, where 3 cases had the calcification located in the center of the lesions and revealed irregular circular or scattered punctate (Fig. 6).

Due to the expansion growth, a fake capsule can be formed around the tumor with an uneven thickness. In the present study, due to the rich blood vessels in the capsule, the capsule was enhanced following an enhanced CT scan, and the boundary was clear (Fig. 5). Capsular invasion and incomplete lesion outlines often indicate malignant lesions. In addition, the cystic components of the tumor always has no enhancement, and the solid components of the tumor can be enhanced early. In the present study, the enhancement was gradual and most evident in the portal or delayed phase. However, the density was close to or still lower than the pancreatic 

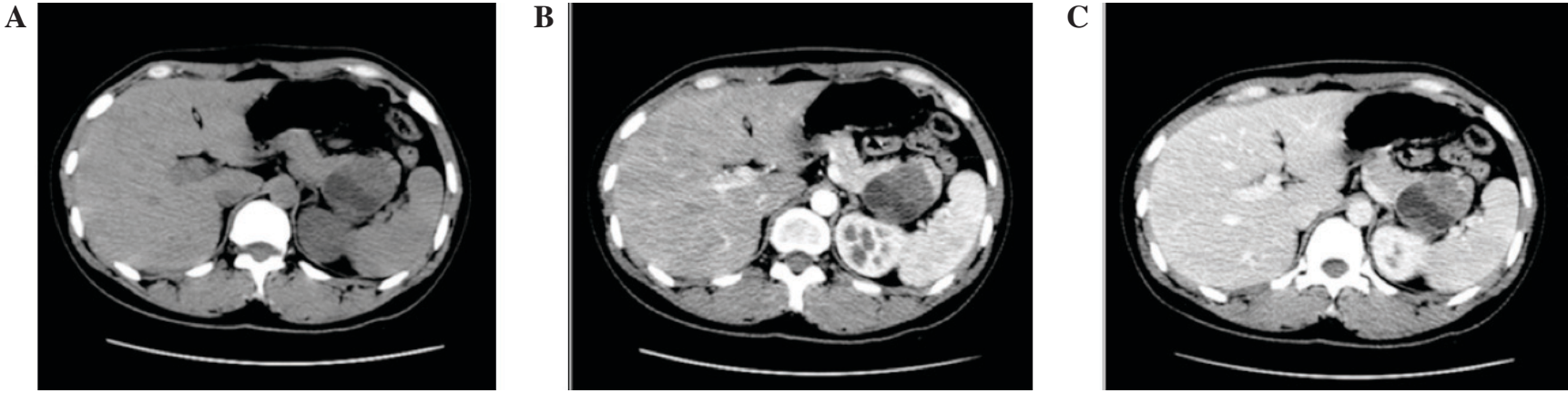

Figure 4. Computed tomography image of solid pseudopapillary tumor of the pancreas of a 31-year old female patient. (A) Tumor tissues were found to be composed by consistent solid-nest tumor cells, and (B and C) the fake papillary structure could be observed.

A

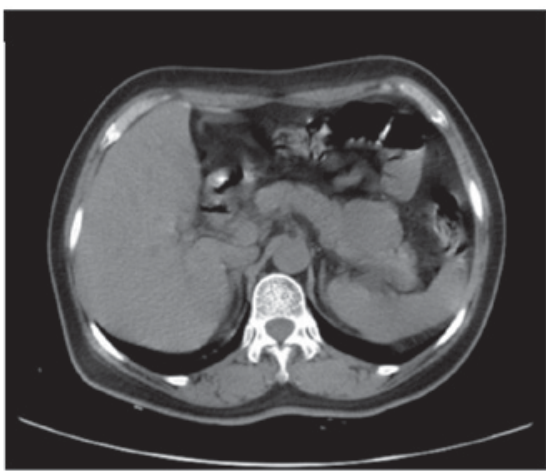

C

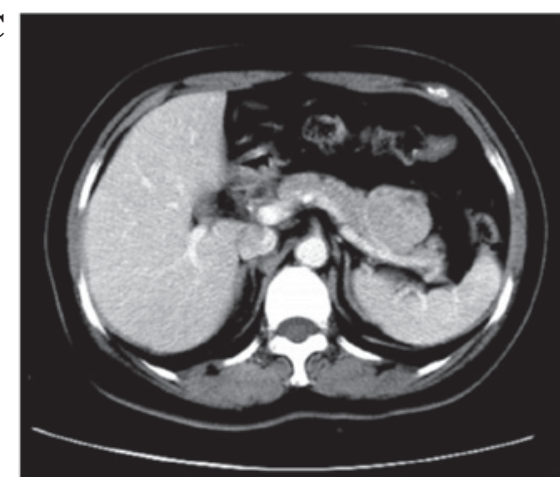

B

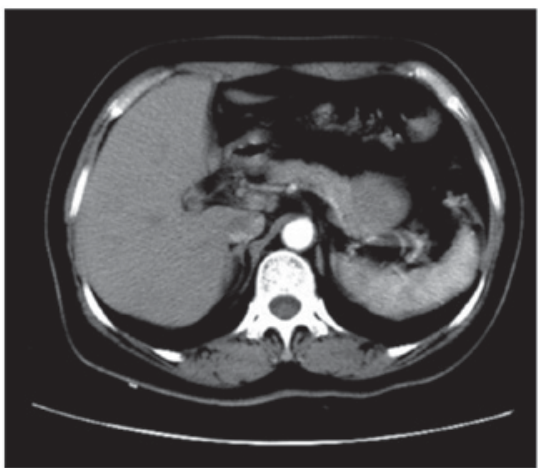

D

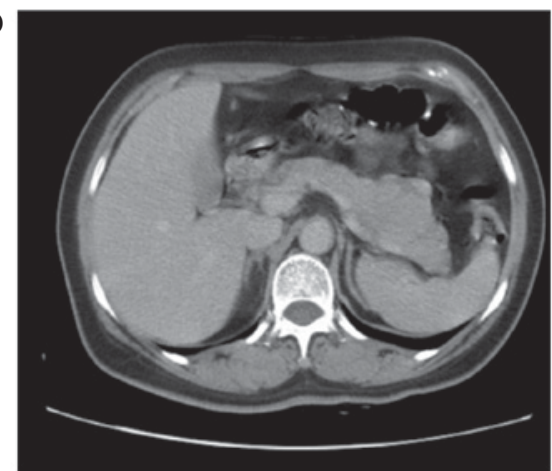

Figure 5. Computed tomograph image of solid pseudopapillary tumor of the pancreas. (A) Scan image showed the boundary is clear, the solid component is the primary component and parts of the lesions are located outside the pancreatic outline. (B) Arterial performance, the enhancement in the arterial phase was uneven, and the density was lower than the pancreas. (C) Portal phase scanning showed the enhancement is significant. (D) Extended scanning showed that lesions were further enhanced, and the density was close to the normal pancreas, unenhanced area could be observed in the solid areas.

parenchyma (Figs. 4 and 5). In the current study, the tumor specimens of the solids were collected for pathological examination, and it was revealed that a large number of collagen fibers and hyaline degeneration could be observed in the tumor stroma (Fig. 2). The existence of such fibrous tissue may be the pathological basis of a delayed enhancement. Due to the slow diffusion of the contrast agent between fibrous stroma tissue and blood vessel (namely the slow process of the contrast agent going through the blood vessels into the fibrous tissue stroma), and it was removed slowly from fibrous tissue stroma to blood vessels, and this may lead to a delayed enhancement phenomenon. Parenchymal necrosis and cystic area was not the strengthen area.

CT manifestations of SPTP have a number of characteristics. These can be described as follows: i) Lesions are predominantly solid and cystic, and cystic and solid components account for different proportions; ii) lesions can be located in any part of the pancreas, often protruding beyond the contour; iii) lesions mostly have clear boundaries with a round, oval and lobulated enhanced capsule observed following CT enhancement; iv) due to the internal hemorrhage and necrosis of the solid part, a plain CT scan shows an uneven density, and the enhancement area can not be observed after an enhanced CT scan; v) following the enhancement of the solid part, different degrees of enhancement can be observed in the arterial phase, which then gradually strengthen to a significant extent, and the strengthening in the portal and delayed phases is even more evident with most parts being lower than the density of the pancreas, and parts close to the normal density of the pancreas; vi) calcification can be observed in the lesion, and the majority of the calcification is located in the edge of the lesion with a small part located in the solid parts of the 

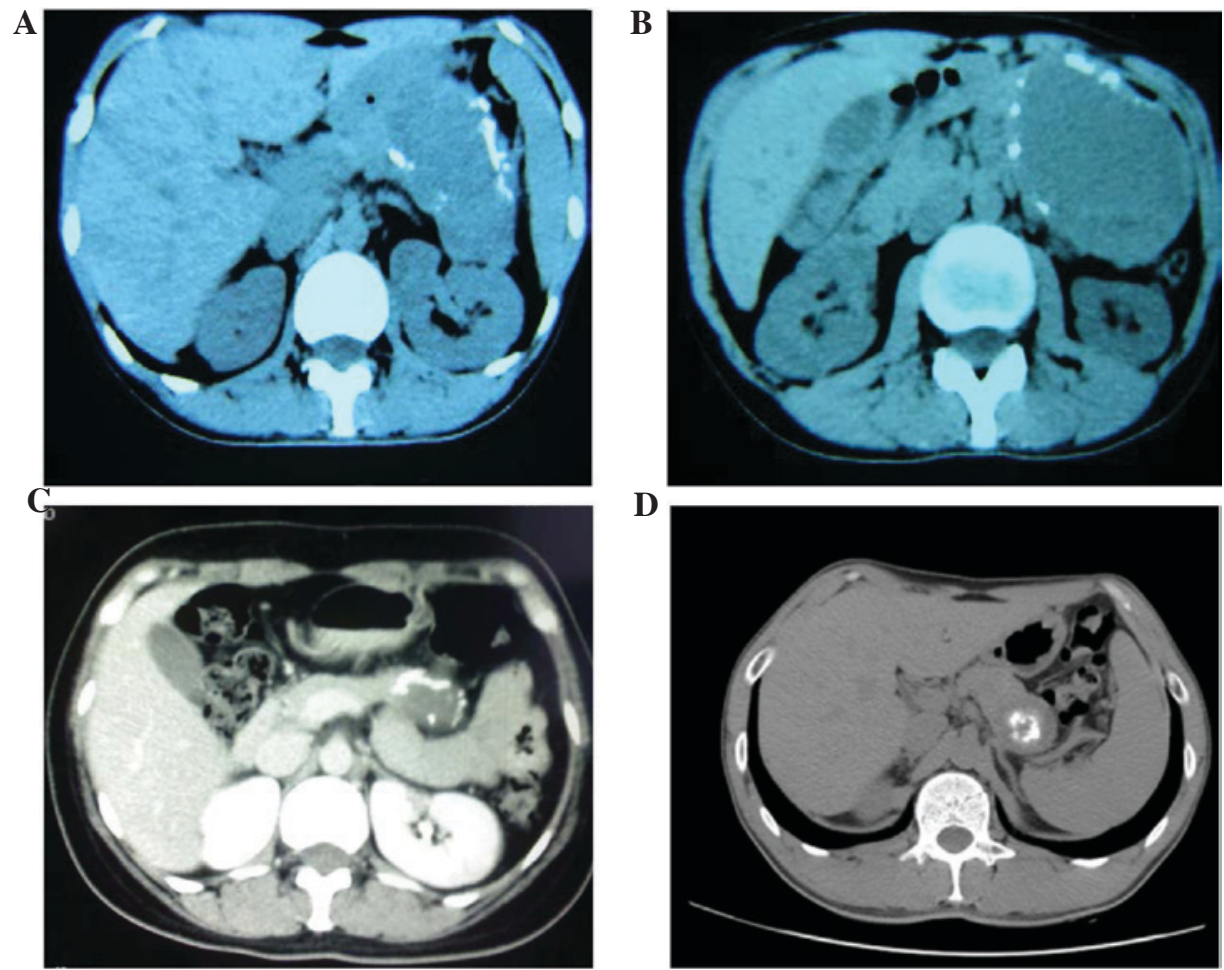

Figure 6. Calcification features of solid pseudopapillary tumor of the pancreas. (A-C) Calcification lesions are located in the edge of the lesion, and are presented as linear arc or multiple punctate calcification, with a few calcifications located in the (D) lesion center, showing irregular circular or scattered dots.

lesion; and vii) no pancreatic duct dilatation and distal pancreatic atrophy is observed.

In conclusion, the onset age of pancreatic cancer is higher and more common in male patients compared with females. Furthermore, invasive growth is often expressed as a solid mass with an unclear boundary. An uneven density is observed, and the majority are located in the outline of the pancreas. In addition, pancreatic cancer is often accompanied by pancreatic duct dilation and distal pancreatic atrophy with calcification being rare, and the surrounding invasion leading to peripancreatic fat gap disappearance. Moreover, liver and lymph node metastasis can be observed.

Pancreas adenomas are common in older females compared with younger ones. Cystic components are the primary components, and are often expressed as multilocular cystic structures, where calcification can be observed in the capsule fibrous septa, and radial calcification is the feature. Furthermore, there is an uneven wall thickness, and an enhanced CT scan demonstrates no delayed enhancement features. In addition, pancreatic duct dilatation can be observed.

The majority of pancreatic endocrine tumors are expressed as a solid mass, round or oval with a clear boundary, and the majority are located in the pancreatic outline. However, there may also be necrosis and cystic degeneration observed, and clinical manifestations of endocrine changes may also be identified.

Finally, the majority of patients with pancreatic pseudocyst have a history of pancreatitis without lobulated change, the capsule wall is thin and uniform, the separating is rare, and early enhancement can be observed after an enhanced CT scan.

\section{References}

1. Crawford BE II: Solid and papillary epithelial neoplasm of the pancreas, diagnosis by cytology. South Med J 91: 973-977, 1998

2. Klimstra DS, Wenig BM and Heffess CS: Solid-pseudopapillary tumor of the pancreas: A typically cystic carcinoma of low malignant potential. Semin Diagn Pathol 17: 66-80, 2000.

3. Adsay NV and Klimstra DS: Cystic forms of typically solid pancreatic tumors. Semin Diagn Pathol 17: 81-8, 2000.

4. Solcia E, Capella C and Kloppel G: Tumors of the exocrine pancreas: Tumors of the Pancreas. In: Atlas of Tumor Pathology. Third Series, Fascicle 20. Armed Forces Institute of Pathology, Washington DC, pp31-144, 1997.

5. Choi JY, Kim MJ, Kim JH, Kim SH, Lim JS, Oh YT, Chung JJ, Yoo HS, Lee JT and Kim KW: Solid pseudopapillary tumor of the pancreas: Typical and atypical manifestations. AJR Am J Roentgenol 187: W178-W186, 2006.

6. Baek JH, Lee JM, Kim SH, Kim SJ, Kim SH, Lee JY, Han JK and Choi BI: Small $(<\mathrm{or}=3 \mathrm{~cm})$ solid pseudopapillary tumors of the pancreas at multiphasic multidetector CT. Radiology 257: 97-106, 2010

7. Cantisani V, Mortele KJ, Levy A, Glickman JN, Ricci P, Passariello R, Ros PR and Silverman SG: MR imaging features of solid pseudopapillary tumor of the pancreas in adult and pediatric patients. AJR Am J Roentgenol 181: 395-401, 2003.

8. Yu PF, Hu ZH, Wang XB, Guo JM, Cheng XD, Zhang YL and $\mathrm{Xu}$ Q: Solid pseudopapillary tumor of the pancreas: A review of 553 cases in Chinese literature. World J Gastroenterol 16: 1209-1214, 2010.

9. Ansari D, Elebro J, Tingstedt B, Ygland E, Fabricius M, Andersson B and Andersson R: Single-institution experience with solid pseudopapillary neoplasm of the pancreas. Scand J Gastroenterol 46: 1492-1497, 2011.

10. Frantz VK: Papillary tumors of the pancreas: Benign or malignant? Tumors of the pancreas. In: Atlas of Tumor Pathology. Section 7, Fascicles 27 and 28. Armed Forces Institute of Pathology, Washington DC, pp32-33, 1959.

11. Vijay A, Ram L, Mathew RK and Chawdhery MZ: Solid pseudopapillary tumor of the pancreas in a patient with cervical cancer: Relation of E-cadherin/ $\beta$-catenin adhesion complex in their carcinogenesis. J Surg Case Rep 2015: rjv034, 2015. 
12. Kloppel G, Solcia E and Longnecker DS: Histological typing of tumors of the exocrine pancreas. In: World Health Organization International Histological Classification of Rumors. Sobin LG (ed). Springer, Berlin, pp15-22, 1996.

13. Papavramidis T and Papavramidis S: Solid pseudopapillary tumors of the pancreas: Review of 718 patients reported in english literature. J Am Coll Surg 200: 965-972, 2005.

14. Cecka F, Jon B, Subrt Z and Ferko A: Solid pseudopapillary tumour of the pancreas: Diagnosis, treatment and prognosis. Acta Chir Belg 114: 58-62, 2014.

15. El Nakeeb A, Abdel Wahab M, Elkashef WF, Azer M and Kandil T: Solid pseudopapillary tumour of the pancreas: Incidence, prognosis and outcome of surgery (single center experience). Int J Surg 11: 447-457, 2013.

16. Peng CH, Chen DF, Zhou GW, Yang WP, Tao ZY, Lei RQ, Zhange SD and Li HW: The solid-pseudopapillary tumor of pancreas: The clinical characteristics and surgical treatment. J Surg Res 131: 276-282, 2006.
17. Buetow PC, Buck JL, Pantongrag-Brown L, Beck KG, Ros PR and Adair CF: Solid and papillary epithelial neoplasm of the pancreas: Imaging-pathologic correlation on 56 cases. J Radiology 199: 707-711, 1996.

18. Vargas-Serrano B, Domínguez-Ferreras E and Chinchón-Espino D: Four cases of solid pseudopapillary tumor of pancreas: Imaging findings and pathological correlations. Eur J Radiol 58: 132-139, 2006

19. Sperti C, Berselli M, Paasquali C, Pastorelli D and Pedrazzoli S: Aggressive behavior of solid-pseudopapillary tumor of pancreas in adults: A case report and review of the literature. World J Gastroenterol 14: 960-965, 2008. 